\title{
Schizophrenia and Parkinson 's disease as Risk Factors for Melanoma Development and Progression!
}

\author{
Valeri Malev ${ }^{1}$, Georgi Tchernev ${ }^{*}$
}

${ }^{1,2}$ Onkoderma-Clinic for Dermatology, Venereology and Dermatologic Surgery, General Skobelev, Sofia,Bulgaria

Received: August 03, 2020; Accepted: August 04, 2020; Published: August 07, 2019

*Corresponding author: Professor Georgi Tchernev, PhD, Onkoderma-Clinic for Dermatology, Venereology and Dermatologic Surgery, Sofia, Bulgaria, General Skobelev Nr 26, Tel. No: 00359885588424; E-mail: georgi_tchernev@yahoo.de

\section{Keywords: Melanoma Development, Melanoma Progression, Schizophrenia, Dopamin, Parkinson}

We present a 39-year-old male patient with skin tumor, localized on the scalp, paranoid schizophrenia and an idiopathic aplastic anemia. The patient presents with a lesion in Regio occipitalis, which occurred a few years ago and progressively enlarged to a big exophytic tumor, causing pain and occasional bleeding (Figure 1a-d). The patient has been diagnosed with a paranoid schizophrenia more than ten years ago, and since then is on therapy with Amisulpride 400mg/d and Haloperidol 2x1,5 mg/d. He does not follow his therapy strictly, but hospitalization has not been necessary.

In 2016 the patient has been diagnosed with idiopathic aplastic anemia and since then is on immunosuppressive therapy with Cyclosporine A $2 \times 100 \mathrm{mg} / \mathrm{d}$. During the dermatological examination we established a well demarcated, $4 \times 4 \mathrm{~cm}$, partly pigmented, exophytic tumor in Regio occipitalis with severe perilesional erythema. The lesion had erosive and necrotic areas and was covered by serous and hemorrhagic crusts with different size (Figure 1a-d). A severe and painful, cervical and occipital lymphadenomegaly was
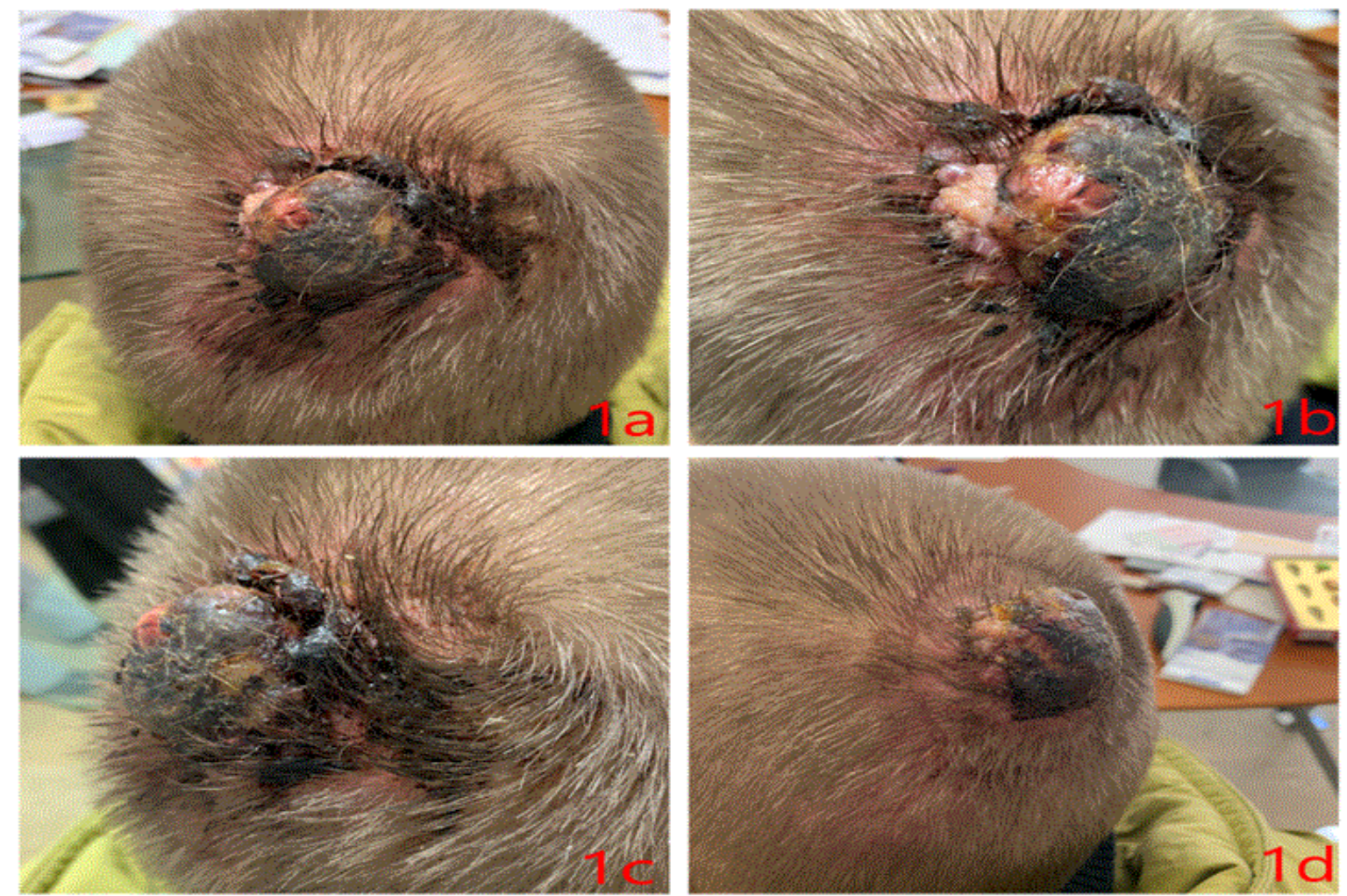

Figures 1a-d: Occipital exophytic tumor with erosive surface and necrotic areas 
to be palpated. The cytologic and histopathologic examinations suggested strongly a malignant melanoma (MM), positive for BRAF mutation. The Magnetic Resonance Imaging (MRI) showed occipital, skin formation with size $4 \times 4 \mathrm{~cm}$ and enlarged, unmatted cervical and occipital lymph nodes. A head Computed Tomography (CT) was performed to exclude skull involvement. The Positron Emission Tomography - Computed Tomography (PET/CT) also suggested a generalized, oncologic disease with multiple metastasis in bones, lymph nodes and spleen, without skull involvement. The final diagnosis was advanced MM in fourth clinical stage. A systemic therapy with Pembrolizumab was started.

A double hemotransfusion was performed due to the critical anemia and thrombocytopenia. A surgical excision of the primal lesion was planned and was later postponed on behalf of patients and his relatives' will.

The critical general condition and disseminated metastasis led to exitus lethalis 3 months after initial diagnosis.

Cutaneous melanoma and paranoid schizophrenia are two socially significant, multifactorial diseases, which share a common pathogenic mechanism [1]. A defect in glutamatergic signal transmitting plays an important role in developing of neuropsychiatric disorders like schizophrenia and Alzheimer's disease, but also in developing of neoplasms like glioma and malignant melanoma [1]. This relation is confirmed by the fact that both group of diseases are positively influenced by the same group of medication [1]. And since in schizophrenia the antidopaminergic therapy leads to clinical improvement and better quality of life, the positive effect in MM is still predominantly scientific and experimental [1]. Back in 1982 it was proved that the dopamine antagonists, used in treatment of schizophrenia, have inhibiting effect on melanoma cell lines [2]. Also proved is the tumoricidal activity of the antipsychotic drug pimozide (3), which is given to its antidopaminergic effect [3]. If partial inhibition of the dopaminergic system has a potential antitumor impact, then we can hypothesize that its increased activity (like in schizophrenia) has a carcinogenic effect, regarding the MM for example [1].

This theory is supported by another strong link between dopaminergic system and cutaneous melanoma - treatment of Parkinson's disease [1]. The prodopaminergic therapy for patients with Parkinsonism is associated with significantly increased risk of cutaneous melanoma [2]. Assigned to the discussed clinical case, the underlying psychiatric disease could be considered as an inducing factor for fast developing of cutaneous melanoma with early locoregional and distant metastasis in a relatively young patient. A significant importance must be given the fact that the patient has not been precise in his antipsychotic medication, which could have had a protective effect, regarding the cutaneous melanoma [1].

Despite that, wide epidemiologic researches with more than 25000 patients with schizophrenia show a decreased risk for oncologic diseases and MM [4]. Regarding the melanoma it could be suggested that this is the effect of the antidopaminergic therapy in patients, who followed their medication regularly [5].

The presented case is of a relatively young patient with advanced melanoma with locoregional and distant metastasis and the underlying psychiatric disease, analogous to other described cases in medical literature, is discussed as an inducing factor for the fast progression of the oncologic disease [1]. The therapeutic approach in patients with advanced melanomas remains a challenge. Various studies with patients in fourth clinical stage of MM show significantly increased survival and 5-year survival rate with combination of metastasectomy and adjuvant target/ immunotherapy, compared to systemic therapy only [5]. This conclusion is valid even for bone metastasis, which are associated with very severe prognosis. Radical metastasectomy increases the overall survival from 4.8 to 11.8 months [6]. Unfortunately, excision of bone metastasis is usually inapplicable, but radical excision of skin, gastrointestinal and lymph metastasis can improve the prognosis [5]. On the other hand, surgical interventions are always associated with more complications like: infections, seromas, fistulation and cicatrisation [5].

Melanoma is a multifactorial disease, which probably shares common pathogenetic mechanisms with paranoid schizophrenia [1]. Radical surgical treatment of primaries and metastasis, could improve the survival rate in a specific group of patients. Every intervention is associated with risk and the approach must be individually adjusted.

To support our statements and their interpretation, more international research is needed to evaluate the association of malignant melanoma and Parkinson's disease, especially in patients treated with dopamine agonists [7-9].

\section{References}

1. Tchernev G, Lozev I, Temelkova I, Chernin S, Yungareva I. Schizophrenia as Potential Trigger for Melanoma Development and Progression! The Psycho Neuro-Endocrine-Oncology (P.N.E.O) Network!. Open Access Maced J Med Sci. 2018;6(8):1442-1445. doi: 10.3889/oamjms.2018.276.

2. Prickett TD, Samuels Y. Molecular pathways: dysregulated glutamatergic signaling pathways in cancer. Clin Cancer Res. 2012;18(16):4240-4246. doi: 10.1158/1078-0432.CCR-11-1217.

3. Elmaci I, Altinoz MA. Targeting the cellular schizophrenia. Likely employment of the antipsychotic agent pimozide in treatment of refractory cancers and glioblastoma. Crit Rev Oncol Hematol. 2018;128:96-109. doi: 10.1016/j.critrevonc.2018.06.004.

4. Foster HD, Hoffer A. Schizophrenia and cancer: the adrenochrome balanced morphism. Med Hypotheses. 2004;62(3):415-9. doi: 10.1016/S0306-9877(03)00319-0.

5. Wollina U, Brzezinski P. The value of metastasectomy in stage IV cutaneous melanoma. Stellenwert der chirurgischen Metastasenentfernung bei Hautmelanomen im Stadium IV. Wien Med Wochenschr. 2019;169(13-14):331-338. doi: 10.1007/s10354-0180630-6.

6. Zekri J, Marples M, Taylor D, Kandukurti K, McParland L, Brown JE. 
Complications of bone metastases from malignant melanoma. J Bone Oncol. 2017;8:13-17. doi: 10.1016/j.jbo.2017.08.003.

7. Ryu HJ, Park JH, Choi M, Jung JH, Han K, Kwon DY, et al. Parkinson's Disease and Skin Cancer Risk: A Nationwide Population-Based Cohort Study in Korea. J Eur Acad Dermatol Venereol. 2020. doi: 10.1111/ jdv.16462.

8. Moriarty N, Moriarty J. Highlighting the Link between Parkinson's
Disease and Malignant Melanoma: A Case Report and Literature Review. Eur J Case Rep Intern Med. 2019;6(11):001297. doi:10.12890/2019_001297.

9. Ye Q Wen Y, Al-Kuwari N, Chen X. Association Between Parkinson's Disease and Melanoma: Putting the Pieces Together. Front Aging Neurosci. 2020;12:60. Published 2020 Mar 10. doi:10.3389/ fnagi.2020.00060 